\title{
Failure detection in robotic arms using statistical modeling, machine learning and hybrid gradient boosting
}

Marcelo Azevedo Costa, Bernhard Wullt, Mikael Norrlöf and Svante Gunnarsson

The self-archived postprint version of this journal article is available at Linköping University Institutional Repository (DiVA):

http://urn.kb.se/resolve?urn=urn:nbn:se:liu:diva-160030

N.B.: When citing this work, cite the original publication.

Costa, M. A., Wullt, B., Norrlöf, M., Gunnarsson, S., (2019), Failure detection in robotic arms using statistical modeling, machine learning and hybrid gradient boosting, Measurement, 146, 425-436.

https://doi.org/10.1016/j.measurement.2019.06.039

Original publication available at:

https://doi.org/10.1016/j.measurement.2019.06.039

Copyright: Elsevier

http://www.elsevier.com/

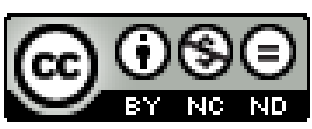




\title{
Failure detection in robotic arms using statistical modeling, machine learning and hybrid gradient boosting
}

\author{
Marcelo Azevedo Costa ${ }^{\mathrm{a}, *}$, Bernhard Wullt ${ }^{\mathrm{c}}$, Mikael Norrlöf ${ }^{\mathrm{c}, \mathrm{b}}$, Svante \\ Gunnarsson $^{\mathrm{b}}$ \\ ${ }^{a}$ Department of Production Engineering, Universidade Federal de Minas Gerais, Brazil \\ ${ }^{b}$ Department of Electrical Engineering, Linköping University, Sweden \\ ${ }^{c}$ Robotics and Discrete Automation, ABB AB, Sweden
}

\begin{abstract}
Modeling and failure prediction are important tasks in many engineering systems. For these tasks, the machine learning literature presents a large variety of models such as classification trees, random forest, artificial neural networks, among others. Standard statistical models such as the logistic regression, linear discriminant analysis, k-nearest neighbors, among others, can be applied. This work evaluates advantages and limitations of statistical and machine learning methods to predict failures in industrial robots. The work is based on data from more than five thousand robots in industrial use. Furthermore, a new approach combining standard statistical and machine learning models, named hybrid gradient boosting, is proposed. Results show that the hybrid gradient boosting achieves significant improvement as compared to statistical and machine learning methods. Furthermore, local joint information has been identified as the main driver for failure detection, whereas failure classification can be improved using additional information from different joints and hybrid models.
\end{abstract}

Keywords: statistical modeling, machine learning, gradient boosting.

\section{Introduction}

In 2001, Leo Breiman published the paper Statistical modeling: The two cultures, describing the two different approaches for data modeling: the data modeling culture and the algorithm modeling culture. The data modeling culture first assumes a parametric, statistical model, hereafter named the

${ }^{*}$ Corresponding author 
white box model, and then uses available data to estimate the parameters of the model and provide statistical inference. The parameters of white box models may have physical meanings. Nonetheless, white box models apply, in general, simple mathematical and statistical structures, such as the linear regression equation. Consequently, the parameters of white box models, also known as coefficients, have meaningful interpretations.

The algorithm modeling culture is mostly interested in finding the most predictive model, hereafter named the black box model, which may have a complex structure and use a large number of variables. In general, black box models comprise general function approximators, which rely on non-linear functions and/or transformations. In general, the parameters of black box models do not have a straightforward interpretation as compared to white box models. However, the parameters of black box models can be tweaked in order to adjust the fitness of the function to different databases. Examples of black box models are neural networks, support vector machines, fuzzy systems, among others.

The basic tool to evaluate prediction performance of both white and black box models is cross validation. According to Breiman et al. (2001), cross-validation is a natural route to the indication of quality of any dataderiving quantity. Briefly, the original data set is divided into two disjoint groups. The first group, named the training set, is used to estimate the parameters of the model. The second group, named the test set, is used to evaluate the error prediction of the previously adjusted model. Models with lower predictive errors are preferable. Finally, it is worth mentioning that Breiman et al. (2001) point out that the best available solution to a data problem may be a data model, or an algorithmic model, or a combination. The data and the problem should guide the solution.

The final application of the present work is failure prediction in industrial robotic arms. According to Ma and Yang (2016) detecting and diagnosing a fault is fundamental to reducing chances of hardware damages or critical injuries to people around controlled systems. Traditionally, monitoring the motion process of robotic arms is performed by analyzing the data collected by sensors attached to the joints or surfaces of the robotic arms (Fan et al., 2017). An alternative approach is to develop dynamic models of the robotic arm and use the residuals of the models to detect faults (Halder and Sarkar, 2009; Shahriari-kahkeshi et al., 2015). Using standard statistical quality control theory (Montgomery, 2007), upper and lower thresholds for the residuals can be estimated in order to detect faults. Interestingly, Fan et al. (2017) proposed the use of successive video frames to detect faults. The proposed method combines optical flow, principal component analysis and indepen- 
dent component analysis to detect faults in robotic-arm-based systems. In general, standard statistical, machine learning, and hybrid methods have been applied to detect failures in robotic arms (Khalastchi et al., 2017). Furthermore, as previouly mentioned by Breiman et al. (2001) and recently pointed by Makridakis et al. (2018), machine learning models may achieve accuracy below standard statistical models. Therefore, the use of statistical, machine learning and hybrid models should be carefully evaluated.

This work evaluates a database comprising failures in industrial robots and production variables. Failure data is available for six joints. The goal is twofold: to find main drivers (features) or main predictors of failures, and to estimate the best predictive model. As previously mentioned, the two objectives are, in general, conflicting. On one side, selecting a few components highly correlated to failure is of utmost importance for good maintenance practices. On the other side, predicting failures as accurately as possible may save production resources and avoid unexpected interruptions in production. Standard statistical and machine learning models are proposed and evaluated.

In addition, a hybrid gradient boosting (HGB) method is proposed. HGB first uses a logistic regression model as a base line model. Therefore, it produces a simple statistical model which indicates variable importance. Furthermore, using a statistical framework based on the gradient boosting algorithm (Friedman, 2001), the HGB creates multiple layers of non-linear (black box) models on top of the logistic regression thereby achieving additional accuracy. As a result, the HGB model share both white and black box optimal properties.

Briefly, the HGB algorithm combines the exponential family distribution representation (Nelder and Baker, 1972) and the Gradient Boost algorithm (Friedman, 2001). Consequently, standard regression, population based regression, one- and multiple-class classification problems can be solved using the proposed algorithm. Using the HGB framework, pseudo-responses are created which allow the combination of different methods, thus improving prediction. Consequently, final classifications combine standard statistical and machine learning models. Standard statistical models provide meaningful interpretations and are generally used as base line models, whereas machine learning models further improve prediction. The novelty of the contribution of this work is twofold. First, classification models for failure detection in industrial robotic arms using statistical and machine learning methods are evaluated. Second, the hybrid gradient boosting algorithm is proposed and applied to failure prediction in industrial robotic arms with improved results. 
It is worth mentioning that, due to natural degradation of the production machines, failures will inevitably happen. Possibly, different failures might happen several times. Thus, up to a certain time, which is unknown, all production machines will fail. Therefore, the available database represents a snapshot of the production robots in a specific time frame. Conclusions with respect to failure drivers are, therefore, constrained to the specific time frame in which the data was collected.

Furthermore, industrial robots are primarily designed to operate under pre-defined conditions, i.e., specific speed range, temperature range, among others. Thus, robots operating under critical or non-expected conditions are more likely to fail. Therefore, outlier detection techniques can be applied to data before using white or black box models. Domingues et al. (2018) presents a comparative evaluation of outlier detection algorithms. Results show that the Isolation Forest (Liu et al., 2008) is an excellent method to efficiently identify outliers. Briefly, Isolation Forest is a non-parametric method which estimates data density using random partitions and tree based structures. Therefore, given the data, it is expected that observations located in low density regions are more prone to failure.

The following classification models were evaluated: logistic regression, regularized logistic regression (glmnet), random forests, extreme gradient boosting (xgboot) and neural networks with extreme learning machine training. In addition, HGB models were evaluated by combining logistic, xgboost and random forests. Results show that, for lower proportion of failures, simpler methods such as logistic and glmnet achieve best results. Whereas, for larger proportion of failures, xgboost, random forests and HGB achieve best results.

Due to data confidentiality, results using public data are available in the supplementary material. These results provides further evidence about the performance of the proposed HGB algorithm. In general, the HGB algorithm may outperform pre-existing algorithms and achieve superior predictive results.

This paper is organized as follows. The proposed hybrid gradient boosting algorithm, classification methods and anomaly score analysis are presented in Section 2. Database description and results are presented in Section 3. Discussion and conclusion are presented in Sections 4 and 5, respectively. 


\section{Materials and Methods}

\subsection{Basic concepts and notation}

Following Friedman et al. (2001), let $\mathbf{x}=\left\{x_{1}, \ldots, x_{p}\right\}$ be a vector of $p$ input variables, hereafter named as predictor variables. Given the predictor variables, the objective is to predict an output variable $Y$, hereafter named as the response variable. This problem is called supervised learning since both inputs and output are known in advance. In addition, let $\left\{y_{i}, \mathbf{x}_{i}\right\}_{i=1}^{N}$ be the observed predictor vector and response variables, where $N$ represents the sample size. If the response variable comprises two-level categorical information, for example, $Y=\{A, B\}$, then the problem is also known as a supervised classification problem in which the response variable is regularly coded as a binary variable written as $Y=\{0,1\}$.

For supervised classification problems, the logistic regression model is a standard statistical model. It assumes that the response random variable follows a Bernoulli distribution, $Y \sim \operatorname{Bernoulli}(\mu)$, where $Y \in\{0,1\}$ and $\mu$ is the probability that the random variable $Y$ is equal to one, $P(Y=1)=\mu$. If predictor variables are available, say $x_{1}, \ldots, x_{p}$, then the $\mu$ parameter can be written as a function of a linear predictor using the logistic function:

$$
P\left(Y=1 \mid x_{1}, \ldots, x_{p}\right)=\frac{\exp \left(\beta_{0}+\beta_{1} x_{1}+\cdots+\beta_{p} x_{p}\right)}{1+\exp \left(\beta_{0}+\beta_{1} x_{1}+\cdots+\beta_{p} x_{p}\right)}
$$

where $0<P\left(Y=1 \mid x_{1}, \ldots, x_{p}\right)<1$ and $\beta$ 's are the parameters of the model which are estimated using maximum likelihood. The Bernoulli log-likelihood function is shown in Equation 2.

$$
\log L i k=-\sum_{i=1}^{N}\left[y_{i} \ln \left(1+e^{-\mu_{i}}\right)+\left(1-y_{i}\right) \ln \left(1+e^{+\mu_{i}}\right)\right]
$$

where $\mu_{i}=\frac{\exp \left(\mathbf{x}_{i}^{T} \boldsymbol{\beta}\right)}{1+\exp \left(\mathbf{x}_{i}^{T} \boldsymbol{\beta}\right)}$ and $\mathbf{x}=\left(1, x_{1 i}, \ldots, x_{p i}\right)$ is the predictor vector. Further details about the logistic model are found in Dobson and Barnett (2008) and Nelder and Baker (1972).

In addition, different penalty functions can be applied to the Bernoulli log-likelihood in order to improve prediction. The lasso (least absolute shrinkage and selection operator) method proposed by Tibshirani (1996), known as the $l_{1}$-norm, and the ridge regression method (Hoerl and Kennard, 1970), also known as the $l_{2}$-norm, are the most widely used penalty functions. Zou and Hastie (2005) proposes the elastic net penalty function which combines the $l_{1}$ - and $l_{2}$-norms. The glmnet package (Friedman 
et al., 2010) implements the elastic net penalty and estimates the penalty parameter using cross-validation.

\subsection{The proposed hybrid gradient boosting}

The proposed hybrid gradient boosting (HGB) defines a general framework for boosting a base line model, such as the standard logistic regression model. In order to boost the base line model, non-linear models such as random forests (Breiman, 2001), xgboost (Chen et al., 2018), extreme learning machines (Huang et al., 2006), or combined models can be applied. For instance, random forests or xgboost models can be applied in order to estimate the feature importance (Breiman, 2001) and, therefore, to identify additional variables which are used to improve prediction.

The HGB approach is based on the exponential family distribution used in the generalized linear models (Nelder and Baker, 1972). A random variable $Y$ belongs to the exponential family distribution if its density distribution can be written as

$$
\log f_{Y}(y \mid \theta, \phi)=\frac{y \theta-b(\theta)}{a(\phi)}+c(y, \phi)
$$

where $\theta$ is the canonical parameter, $\phi$ is the dispersion parameter, and $b(\theta)$, $c(y, \phi)$ and $a(\phi)$ are functions related to the density distribution of $Y$. From Equation 3, the log-likelihood function can be written as:

$$
\log L i k=\sum_{i=1}^{N} \frac{y_{i} \theta_{i}-b\left(\theta_{i}\right)}{a(\phi)}+c\left(y_{i}, \phi\right)
$$

The exponential family distribution has very interesting properties, such as $E(Y)=b^{\prime}(\theta)$ and $\operatorname{Var}(Y)=a(\phi) b^{\prime \prime}(\theta)$. In general, a linear predictor can be incorporated into the exponential distribution by assuming $\theta(\mathbf{x})=$ $\mathbf{x}^{T} \boldsymbol{\beta}$, where $\mathbf{x}^{T} \boldsymbol{\beta}=\beta_{0}+\beta_{1} x_{1}+\ldots+\beta_{p} x_{p}$ is the linear predictor. Density distributions such as Normal, Poisson, Binomial, Bernoulli, Multinomial and Gamma can be written using Equation 3. Further details about exponential family distribution and generalized linear models are found in Nelder and Baker (1972), Dobson and Barnett (2008), and elsewhere.

Following Friedman (2001), we propose an additive expansion for the canonical parameter $\theta_{(\mathbf{x})}$, shown in Equation 5 .

$$
\theta_{(\mathbf{x})}=\sum_{m=0}^{M} \theta_{m}\left(\mathbf{x} ; \boldsymbol{\beta}_{m}\right)
$$


For instance, $\theta_{0}\left(\mathbf{x} ; \boldsymbol{\beta}_{0}\right)$ represents the standard linear predictor, i.e., the logistic regression solution; whereas $\theta_{1}\left(\mathbf{x} ; \boldsymbol{\beta}_{1}\right), \ldots, \theta_{M}\left(\mathbf{x} ; \boldsymbol{\beta}_{M}\right)$ may represent different models such as classification and regression trees and neural networks, among others. $\boldsymbol{\beta}_{m}$ represents the vector of parameters associated with each model or boost.

Using the exponential family representation and the additive expansion shown in Equation 5, the proposed HGB algorithm is presented below.

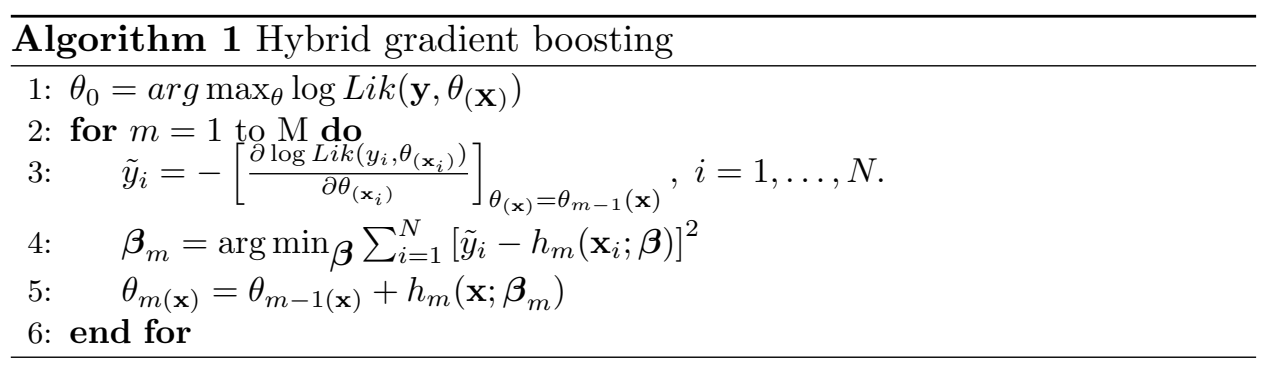

Line 1 represents the base line model, which is adjusted using maximum likelihood. Using the exponential family representation, Line 3 can be written as $\tilde{y}_{i}=y_{i}-E\left(Y_{i} \mid \mathbf{x}_{i}, \theta_{m-1\left(\mathbf{x}_{i}\right)}\right)$. Line 4 shows that the $m$-boost model $h_{m}\left(\mathbf{x}_{i} ; \boldsymbol{\beta}\right)$ is fitted using a least square minimization problem. Therefore, $h_{m}(\mathbf{x} ; \boldsymbol{\beta})$ comprises a non-linear regression model. It is worth mentioning that the additive expansion shown in Equation 5 can generate positive or negative values, as expected using the exponential family. Nonetheless, the estimated mean is $E\left(Y_{i} \mid \mathbf{x}_{i}, \theta_{m\left(\mathbf{x}_{i}\right)}\right)=b^{\prime}\left(\theta_{m\left(\mathbf{x}_{i}\right)}\right)$. For instance, if $Y$ follows a Bernoulli distribution, then $b^{\prime}\left(\theta_{m\left(\mathbf{x}_{i}\right)}\right)$ is the logistic function. The HGB algorithm assuming a Bernoulli distribution, i.e., a classification problem, is shown in Figure 1.

As previously described, the base line model, i.e., the logistic regression, is first used to fit the data. Thus, the estimated coefficients of the logistic model are estimated solving the following maximization function:

$$
\hat{\boldsymbol{\beta}}=\arg \min _{\boldsymbol{\beta}} \log \operatorname{Lik}(\boldsymbol{\beta})
$$

where $\log \operatorname{Lik}(\boldsymbol{\beta})$ is described in Equation 2. The logistic linear predictor is written as $\theta_{0}(\mathbf{x}, \hat{\boldsymbol{\beta}})=\mathbf{x}^{T} \hat{\boldsymbol{\beta}}$ and the model estimated response is

$$
\hat{P}(Y=1)=e^{\theta_{0}(\mathbf{x}, \hat{\boldsymbol{\beta}})} /\left(1+e^{\theta_{0}(\mathbf{x}, \hat{\boldsymbol{\beta}})}\right)
$$

In sequence, the HGB algorithm creates new pseudo-responses, $\tilde{y}_{i}$, which are used as dependent variables in black-box regression problems. For in- 


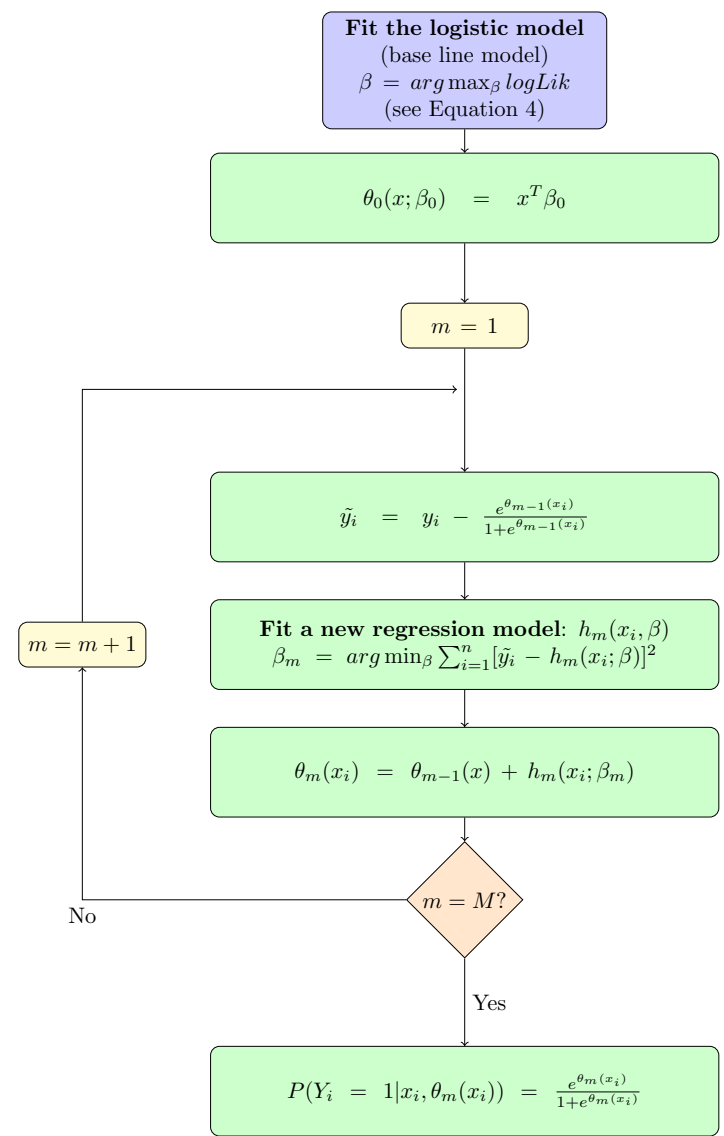

Figure 1: Flowchart of the hybrid gradient boosting (HGB) algorithm.

stance, after estimating the base line model, the pseudo-responses are estimated as

$$
\tilde{y}_{i}=y_{i}-\frac{e^{\theta_{0}\left(\mathbf{x}_{i}, \hat{\boldsymbol{\beta}}\right)}}{1+e^{\theta_{0}\left(\mathbf{x}_{i}, \hat{\boldsymbol{\beta}}\right)}}
$$

Next, in the first step $(m=1)$ of the HGB algorithm, the parameters of the first black-box model are estimated using minimum least squares and the pseudo-responses, as shown in Equation 9.

$$
\hat{\boldsymbol{\beta}}_{1}=\arg \min _{\boldsymbol{\beta}} \sum_{i=1}^{n}\left[\tilde{y}_{i}-h_{1}\left(\mathbf{x}_{i} ; \boldsymbol{\beta}\right)\right]
$$


where $h_{1}\left(\mathbf{x}_{i} ; \boldsymbol{\beta}_{1}\right)$ is the black-box regression equation and $\boldsymbol{\beta}_{1}$ are the respective parameters. Using the estimated response of the black-box model, the logistic linear predictor, i.e., the canonical parameter, is updated with the estimated black-box response, as shown in Equation 10.

$$
\theta_{1}\left(\mathbf{x}_{i}\right)=\theta_{0}\left(\mathbf{x}_{i}\right)+h_{1}\left(\mathbf{x}_{i} ; \hat{\boldsymbol{\beta}}_{1}\right)
$$

The new response of the boosted base-line model is:

$$
\hat{P}(Y=1)=e^{\theta_{1}\left(\mathbf{x}_{i}\right)} /\left(1+e^{\theta_{1}\left(\mathbf{x}_{i}\right)}\right)
$$

At each new step $(m \geq 2)$ of the HGB algorithm, new pseudo-responses are created based on the differences between observed and accumulated responses of previous layers of models. Therefore, each new pseudo-response carries the information not captured by previous layers. Furthermore, minimum least squares are applied to fit the new black box models, $h_{m}\left(\mathbf{x}_{i} ; \boldsymbol{\beta}_{m}\right)$, to the new pseudo-responses. Thus, $\hat{\boldsymbol{\beta}}_{m}$ are the estimated parameters of the black-box in the $\mathrm{m}$-th step. Using the exponential family representation, the estimated responses of the black-box models are accumulated in the canonical parameter $\theta_{m(\mathbf{x})}$. The concept is similar to xgboost but combining different models using a generalized statistical structure.

For regression problems, the HGB algorithm creates successive layers of models by using the residuals of the previous layers as new pseudo-responses for the upcoming layers.

\subsection{Classification models}

The proposed model combines different classification methods by boosting a base line model using different classification methods. In the case study, the logistic regression model, described in section 2.1, is used as the base line model. Random forests, neural networks with extreme learning machine algorithm, and xgboost models were used to boost the base line model.

Random forests (Breiman, 2001) are combinations of CART (Classification And Regression Tree) models. The classification tree model is a local mean model which creates partitions of the original data and estimates $P(Y=1)$ using the local mean, $\bar{y}$ (Murthy, 1998; Breiman, 2017). Each tree or CART model is adjusted using a random sample of the predictors. The outcome of the Random Forests is a combination of the outcome of each CART model. Either, the mean or the median statistic of the $n$ CART models are the most common aggregation statistics. In addition, the average contribution to the maximization of the likelihood for each predictor 
is calculated. This statistic, known as the feature importance, is used to identify the most important predictors.

Neural networks are also popular black box models. Neural networks are universal approximators. It has been shown that feed forward networks with a single hidden layer and with a finite number of neurons can approximate continuous functions (Gybenko, 1989). Nevertheless, neural network training may be time consuming. Huang et al. (2006) proposes the extreme learning machine (elm) algorithm for single-hidden layer feed forward neural networks. The algorithm creates random values for the input weights of the neural networks. The remaining parameters of the neural network are estimated using minimum least squares. The elm neural network requires more hidden neurons as compared to neural networks using standard gradient based algorithms. In addition, standard regularization techniques for linear models, such as the elasticnet (Zou and Hastie, 2005) can be combined with the elm algorithm. Huang et al. (2006) shows that elm achieves much faster convergence of the neural network as compared to standard neural network training algorithms.

The eXtreme Gradient Boosting, or xgboost, is a fast implementation of the gradient boosting decision tree algorithm (Friedman, 2001) and, recently, has been largely applied to classification problems. xgboost uses more regularized model formalization to control overfitting, which gives it better performance. xgboost (Chen et al., 2018) proposes a general framework for the gradient boost algorithm in order to improve optimization. The basic idea is to take the Taylor expansion of the loss function up to the second order and, therefore, approximate the minimization of the loss function as a minimum squared error problem. In addition, the objective function includes a regularization term, or a model complexity term, thus creating a score structure. The score structure is used to optimize the size of classification trees, which is the standard classification model used by xgboost. Consequently, using xgboost, feature importance statistics are also estimated for each predictor. Further details about xgboost are found in Chen et al. (2018) and Friedman (2001).

\subsection{Isolation Tree and Isolation Forest}

Although isolation trees (iTree) and CART models are based on binary trees, the iTree (Liu et al., 2008) aims at detecting data anomalies using an unsupervised approach, i.e., there is no response variable $Y$. The iTree algorithm creates binary trees by randomly choosing $x$ variables, or features, and randomly choosing thresholds $\tau$. The iTree algorithm requires one parameter which is the maximum height of the tree $l$. Briefly, the algorithm 
randomly creates partitions in the data until the maximum height parameter of the tree is reached, or stops earlier if, in each leaf of the tree, there is only one observation. Similar to random forests, a set of isolation trees is created by randomly selecting subsets of data and fitting a new iTree to each subset. After creating a large number of iTrees, hereafter known as isolation Forest, or simply iForest, the complete database is presented to each iTree. For each observation, the path length for each iTree is estimated and a final anomaly statistic is calculated by averaging the path length among the iTrees. Anomalies are those observations with short average path lengths on the iTrees. Furthermore, Liu et al. (2008) proposes an anomaly score based on the average path length. The anomaly score lies between zero and one. If the anomaly score is greater than 0.6 then the observation is classified as an anomalous observation. It is worth mentioning that Domingues et al. (2018) claims that iForest is an efficient method to identify outliers.

The iForest has been applied in anomaly detection applications (Puggini and McLoone, 2018). However, as opposed to detect anomalous data, the present work proposes the use of the anomaly score as a new predictor variable for failure detection. This is because, in failure detection problems, observations located in low density regions may represent observations under extreme conditions and, therefore, more likely to failure.

\subsection{Cross validation, sensitivity and specificity analysis}

Prediction accuracy of the proposed statistical and machine learning models was estimated using a 10-fold cross validation procedure. Initially, the original database is randomly partitioned into 10 disjoint folds. In sequence, the database and the respective partitions are replicated 10 times. Each replicate represents the original database. For each replicate, one fold is the test set and the remaining 9 folds are the training set. Therefore, 10 models are adjusted and 10 predictions are generated. The resulting prediction for each fold is combined into a new database, which is exactly the same size as the original data. From the predicted database, a final test statistic, or prediction statistic, is calculated. In order to account for the randomness of the 10-fold partition process, the entire procedure is repeated, say 10 times, and the average prediction statistic is evaluated.

Sensitivity and specificity statistics are widely applied in medical tests (Altman, 1994). In binary classification tests, these statistics are used as performance measures (Christopher, 2011). Sensitivity, also known as true positive rate, measures the proportion of observed positive classes $\left(y_{i}=1\right)$ which is correctly identified by the classification model. Specificity, also known as true negative rate, measures the proportion of observed negative 
classes $\left(y_{i}=0\right)$ which is correctly identified by the classification model. Thus, sensitivity and specificity are performance measures related to observed positive and negative classes, respectively.

In general, statistical classification models and machine learning models estimate the probability of a new observation being classified as a positive class given a vector of predictors, $0<\hat{P}\left(Y=1 \mid x_{1}, \ldots, x_{p}\right)<1$, the analyst must decide whether the event $Y=1$ will eventually happen. Thus, a threshold $\tau$ is selected and the decision rule, shown in Equation 12, is applied.

$$
\hat{y}_{0}= \begin{cases}1, & \text { if } \hat{P}\left(Y=1 \mid \mathbf{x}_{0}\right) \geq \tau \\ 0, & \text { if } \hat{P}\left(Y=1 \mid \mathbf{x}_{0}\right)<\tau\end{cases}
$$

Both sensitivity and specificity statistics are affected by threshold $\tau$. Consequently, there is an optimal value of $\tau^{*}$ in which sensitivity $=$ specificity. Furthermore, the receiver operating characteristic (ROC) curve (Bradley, 1997; Fawcett, 2004) summarizes the performance of the classification model for different values of $\tau$. The area under the ROC curve (AUC area under the curve) is widely used as a classification performance statistic. Optimal classification models have large AUC. In addition to the AUC, this work proposes the use of the intersection point between sensitivity and specificity, i.e., the $\tau^{*}$ threshold, as a classification performance statistic, hereafter named as classification rate.

\section{Results}

\section{1. $A B B$ robot data}

$\mathrm{ABB}$ robotics is a leading supplier of industrial robots. The ABB data available for the analysis comprise approximately 6,000 observations, one observation per unique robot. For each robot, data for the movements of the six joints are available. In total, 275 variables are available in the database for each robot. Variables can be categorized into two major groups: (a) operational features and (b) robot type. Variables in the operational features group can be further divided into joint operational features and robot operational features. For each joint, the following information is available: average speed (avg_speed), average torque (avg_torque), accumulated feature combining angle and speed (angle_speed_comb), accumulated absolute position (moved_distance), number of emergency stops (nr_E_stops), run time in hours (r_time), wait time in hours (w_time) and failed information (has_failed). Run time comprises the accumulated time in which the robot 
joint presented a non-zero speed. Wait time comprises the accumulated time in which the robot joint is controlled in a steady state with no mechanical brake applied. Combining angle and speed comprises an accumulated feature computed as a nonlinear combination of angle and speed. Larger values of angle and speed are the main contributors to the estimation of this feature. This variable was originally developed as an input to degradation models, proposed by the robot manufacturer. Unfortunately, further information regarding the exact computation of this variable cannot be provided due to data confidentiality.

The failed information comprises the presence $(y=1)$ or absence $(y=0)$ of failure in each joint. One type of fault, related to the degradation of one specific component found in all axis was investigated. It is worth mentioning that the data do not represent random samples from the total fleet of robots. Therefore, the observed proportion of failures may be different if another set of data is collected from a different group of robots.

Figure 2 illustrates the robotic arm and indicates the six joints. Joint 1 comprises the rotating base of the robot. Joint 2 comprises the horizontal movement of the arm set. Joint 3 is known as the elbow and comprises the vertical movement of the arm. Joint 4 comprises the forearm rotation movement. Joint 5 comprises the wrist movement of the robotic arm and joint 6 comprises the movement of the hand tool, which may be a welding tool, grip tool, among others.

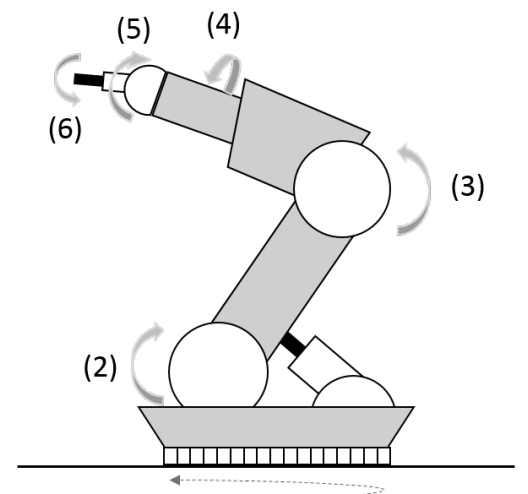

(1)

Figure 2: Configuration of the robotic arm.

Figure 3 illustrates the pairwise correlation between joint operational features (or variables). The narrower the ellipse the larger the correlation. 
Blue ellipses have positive slopes and indicate positive correlations, whereas red ellipses have negative slopes and indicate negative correlations. White circles indicate weak correlations. Figure 3 shows clusters of correlated variables in the center and at the top of the figure.

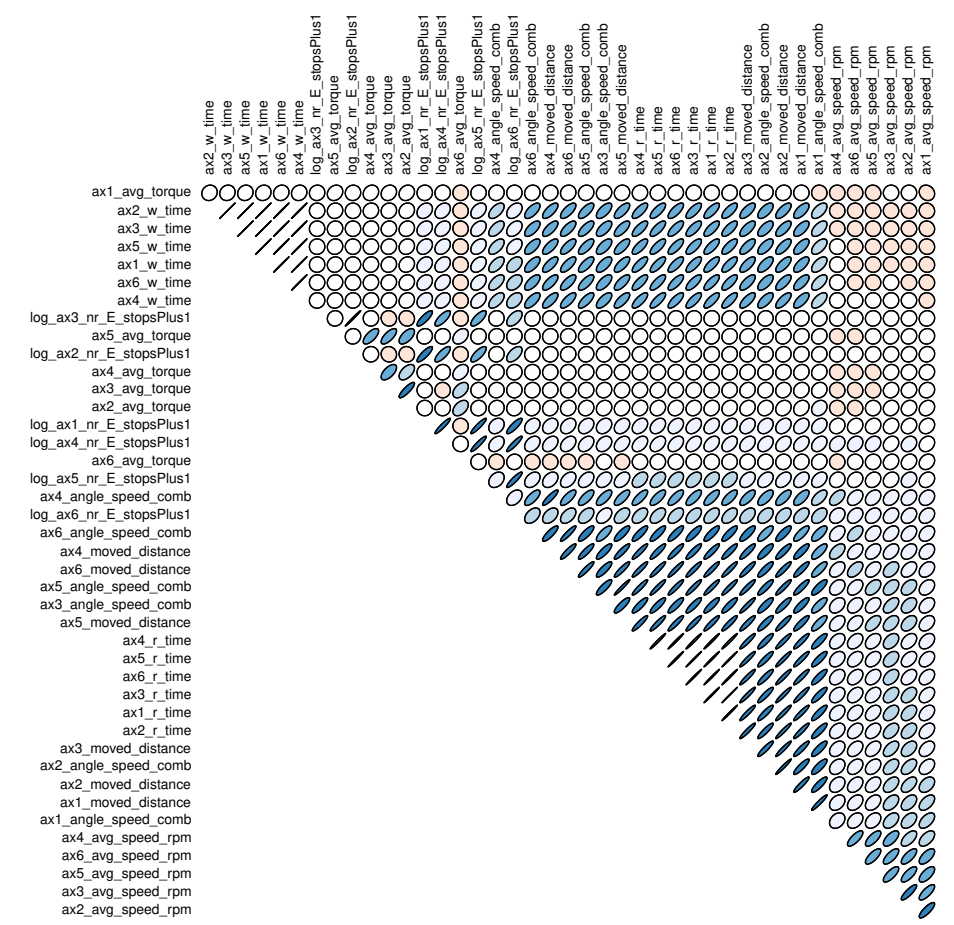

Figure 3: Correlation matrix using average torque, run time, average speed, combined angular position and speed, number of emergency stops, moved distances and wait time for joints 1 to 6 .

The robot operational features comprise the following variables: time when the system was first started (date_start), time of the last service (date_service), production time in hours (p_time), robot type (r_type) and accumulated energy consumption (sys_energy). A new variable was created by dividing the accumulated energy consumption by the production time. This variable, named SysDivPtime, represents the energy consumption rate by production hour.

In addition to the joint variables, previously mentioned, histogram information for each joint is also available. This information represents sequential measures for angles, torque and speed; it comprises relative frequen- 
cies in pre-specified range intervals, or bins. For instance, suppose speed information is available for joint 1 . The variable ax1_avg_speed is the average speed. The entire range of observed values for ax1_speed are divided into a series of adjacent and non-overlapping intervals (bins). Therefore, ax1_hist_speed_bin_0 is the lower bound of the observed relative frequency of speed in joint 1 . There are 216 histogram variables in the database. Figure 4 illustrates the histogram variables for observed speed and angle in joint 1. For each bin, the relative frequency comprises one separate variable. The bins with the largest relative values comprise, in general, standard operating conditions.

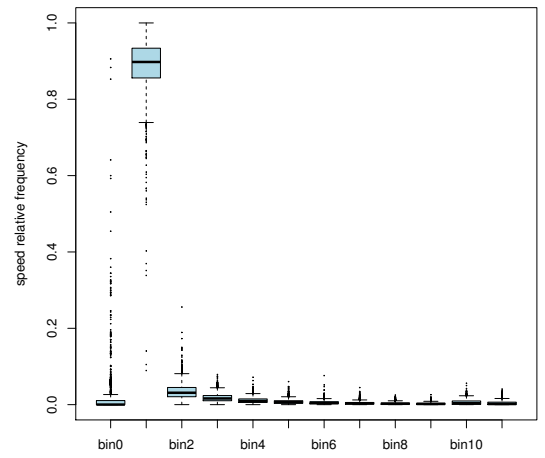

(a) Speed histogram information.

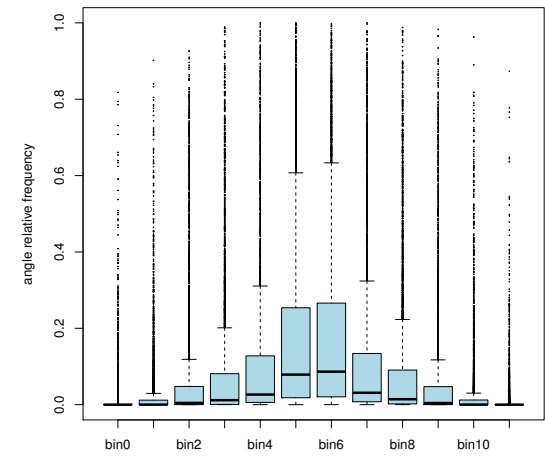

(b) Angle histogram information

Figure 4: Speed and angle histogram information for joint 1.

The proportion of failure for each joint is $0.2 \%$ for joint $1,1.1 \%$ for joint $2,1.6 \%$ for joint $3,6.3 \%$ for joint $4,2.4 \%$ for joint 5 and $5.7 \%$ for joint 6. Joints 4 and 6 present the largest proportion of failures as compared to the remaining joints in the database. Each observation, i.e., each robot, can be classified into one of 5 different robot type categories. $58.9 \%$ of the observations comprise robot type $1,33.1 \%$ comprise robot type $2,7.9 \%$ comprise robot type 3 and the few remaining observations comprise robot types 4 or 5. Empirical analysis, using the proportion of failure for each robot type, indicates the robot type as a potential predictor variable, which is included as a dummy variable.

Data consistency analysis identified a few negative observations for the number of emergency stops. Negative values were replaced by missing data (NA Not Available). In addition, the number of emergency stops presented 
heavy-tailed empirical distributions with many extreme values. Thus, the logarithm of the number of emergency stops plus one was used as the new predictor variable, as opposed to the raw number of emergency stops.

\subsection{Proposed classification models}

Six classification models are initially proposed: the standard logistic regression model (logistic); the logistic regression model using elasticnet penalty (glmnet); two neural network models with 75 and 350 hidden nodes, respectively, using a combined elm and elastic net algorithm (glmNNET75 and glmNNET350); the random forests model (RandomForest); and, the extreme gradient boosting model (xgboost).

The anomaly score is estimated using only local joint production variables, i.e., average speed in revolutions per minute (avg_speed_rpm), average torque (avg_torque), accumulated feature combining angle and speed (angle_speed_comb), accumulated absolute position (moved_distance), logarithm of the number of emergency stops plus one (log_nr_E_stopsPlus1), run time in hours (r_time) and wait time in hours (w_time). For instance, to estimate a classification model for joint 4 , only local joint production variables are used to calculate the anomaly score. Therefore, separate anomaly scores are calculated for each joint. The anomaly score is sensitive to the number of variables and the number of trees (Liu et al., 2008). Stable anomaly scores are generated using a smaller set of variables and a larger number of trees. The anomaly scores are estimated setting the number of trees equal to 1,000 .

The standard logistic regression model is estimated using a white-box modeling approach. The local joint production variables and the anomaly score are evaluated. Empirical evidence using frequency plots shows that the proportion of failures is larger for robot types 2 and 3. Therefore, two dummy variables are also included in the logistic regression model. Variables are selected based on statistical inference, i.e., the P-value. Variables which are not statistically significant at the $\alpha=0.05$ level are gradually removed from the model. Thus, the final model comprises statistically significant variables.

The glmnet, glmNNET75, glmNNET350, RandomForest and xgboost constitute the black box models. These models are estimated and evaluated using the 10-fold cross validation procedure presented in Section 2.5. Initially, all 276 available predictors, including the anomaly score, are used.

Table 1 presents the estimated logistic regression coefficients using the total database for failure detection in joint 4 . Joint 4 has the largest number of failures $(6.3 \%)$. The local predictors (joint level), the anomaly score 
and the robot types 2 and 3 are evaluated. Results show that average speed, combined angle and speed, number of emergency stops and wait time have positive coefficients, as expected. On the contrary, the anomaly score presents a negative coefficient. Thus, the model indicates that the lower the anomaly score, the larger the chance of a failure. As previously described, the anomaly score represents the density of the data. Larger anomaly scores indicate observations in lower density regions. In an industrial setting, large anomaly scores comprise observations under non-normal operating circumstances. However, the database comprises a time snapshot of the operating robots. It is expected that, eventually, all robots will present failure. Furthermore, the average speed, combined angle and speed, number of emergency stops and wait time also capture non-normal conditions. The anomaly score coefficient points towards the large density region, i.e., the lower anomaly score. This result suggests that some of the failures are randomly scattered in the predictors space. Consequently, the larger the density, the larger the number of failures, as expected.

Table 1: Logistic regression results for failure detection in joint 4.

\begin{tabular}{lrrr}
\hline Coefficient & Estimate & Std. Error & P-value \\
\hline Intercept & -2.3134 & 0.6778 & 0.0000 \\
ax4_avg_speed_rpm & 0.8162 & 0.1519 & 0.0000 \\
ax4_angle_speed_comb & 0.0587 & 0.0161 & 0.0003 \\
log_ax4_nr_E_stopsPlus1 & 0.3137 & 0.0563 & 0.0000 \\
ax4_w_time & 0.2240 & 0.0498 & 0.0000 \\
anomaly_score & -8.9441 & 1.9956 & 0.0000 \\
robot type 2 & 0.3897 & 0.1164 & 0.0008 \\
robot type 3 & 0.5704 & 0.1805 & 0.0016 \\
\hline
\end{tabular}

One may claim that the anomaly score is highly correlated to average speed, combined angle and speed, number of emergency stops and wait time. Therefore, results presented in Table 1 are subject to multicollinearity. Figure 5 shows the correlation plot between the predictors presented in Table 1. Pairwise correlations are represented as ellipses. The narrower the ellipse, the larger the correlation. Figure 8 shows that the anomaly score variable is positively correlated to combined angle and speed $(\rho=0.6531)$ and wait time $(\rho=0.5599)$. Nevertheless, a Variance Inflation Factor (VIF) analysis (Montgomery et al., 2012) shows that the largest VIF statistics are 2.0141 for the anomaly score and 2.8114 for combined angle and speed. In general, a critical value of $5(\mathrm{VIF}>5)$ or $10(\mathrm{VIF}>10)$ is used to indicate strong evidence of multicollinearity among the predictor variables. Therefore, there is no 
evidence of strong correlation between the anomaly score and the evaluated predictors. As previously described, the anomaly score measures the density of the data rather than a linear combination of the predictors. The logistic regression model, using the total number of observations, achieves an AUC of 0.7083 and a classification rate of 0.6473 .

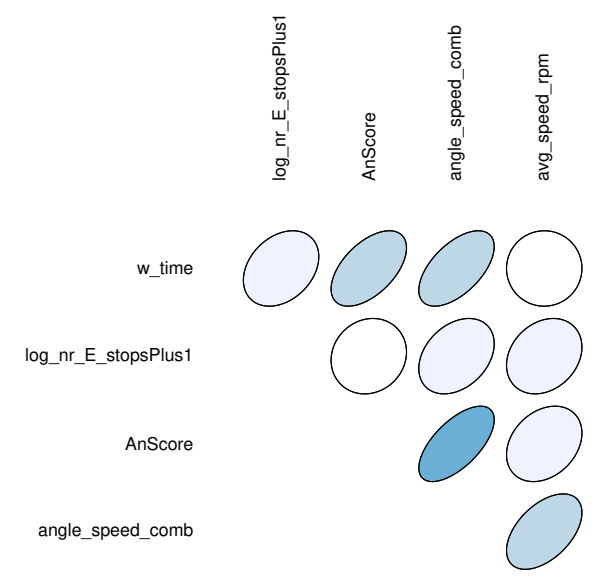

Figure 5: Correlation matrix using average speed, combined angle and speed, number of emergency stops, wait time and anomaly score for joint 4 .

Figure 6 shows the statistically significant predictors, using the logistic regression model for each joint. It can be seen that average speed (avg_speed_rpm) is the common predictor. For joint 1, which has the lowest proportion of failures, only average speed and accumulated absolute position (moved_distance) are statistically significant. Furthermore, for joints 2 to 6 the anomaly score is also a common predictor.

Figure 7(a) shows the boxplot of the AUC statistic for each classification method for joint 4. For comparison purposes, the logistic regression was included in the 10 -fold cross validation analysis using the statistically significant predictors. Results show that xgboost achieves the largest AUC, followed by Random Forests. It is worth mentioning that the logistic regression achieved the lowest standard deviation, thereby achieving the greatest precision. Figure 7(b) shows the boxplot of the classification rate statistic for each classification method. Results also show that xgboost achieves the largest classification rate, followed by glmnet and Random Forests. 


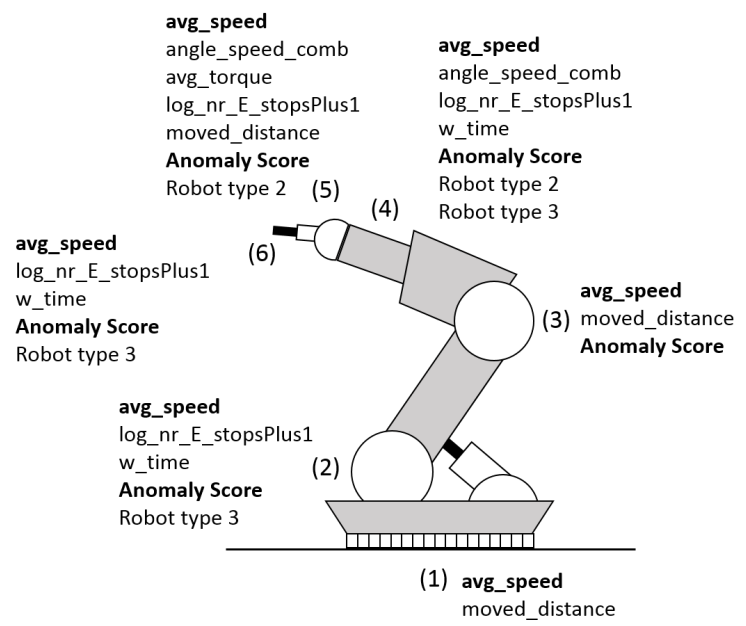

Figure 6: Statistically significant predictors using the Logistic model.

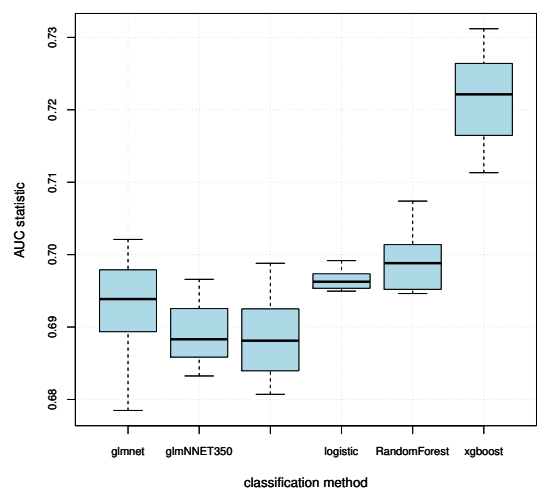

(a)

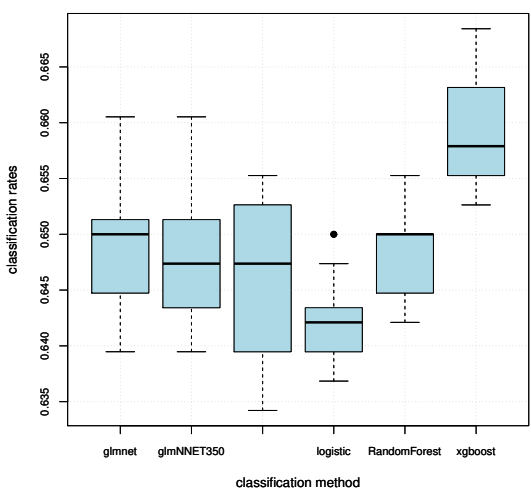

(b)

Figure 7: AUC (Area Under the Curve) statistic (a) and classification rates (b) for failure detection in joint 4 using the selected classification methods in 10 runs.

Table 2 summarizes AUC, classification rates and computing times for all evaluated methods used to predict failure in joint 4 . Best results are shown in bold type. It can be concluded that xgboost achieves the best AUC and classification results, whereas the logistic regression achieves lower AUC standard deviation. Regarding computing time, the logistic regression is the fastest method, followed by xgboost and glmNNET75. 
Table 2: AUC, classification rates and computing times, evaluated in 10 runs of the selected classification methods for failure detection in joint 4 . Best results and minimum standard deviations are indicated as bold type.

\begin{tabular}{lrrrrrr}
\hline \multirow{2}{*}{ method } & \multicolumn{2}{c}{ AUC } & \multicolumn{2}{c}{ Classification rates } & Computing time (min) \\
\cline { 2 - 6 } glmnet & mean & sd & mean & sd & mean & sd \\
glmNNET350 & 0.6929 & 0.0074 & 0.6486 & 0.0064 & 32.2128 & 4.8953 \\
glmNNET75 & 0.6891 & 0.0047 & 0.6481 & 0.0067 & 103.4436 & 184.2138 \\
logistic & 0.6888 & 0.0062 & 0.6459 & 0.0076 & 13.1653 & 2.2524 \\
Random Forests & 0.6965 & $\mathbf{0 . 0 0 1 3}$ & 0.6421 & $\mathbf{0 . 0 0 3 9}$ & $\mathbf{0 . 0 1 3 8}$ & $\mathbf{0 . 0 0 1 2}$ \\
xgboost & 0.6990 & 0.0043 & 0.6486 & 0.0040 & 65.9301 & 14.4743 \\
\hline
\end{tabular}

It is worth comparing the logistic regression results using the whitebox and the black-box approach, i.e., the logistic regression using elasticnet penalty and 10-fold cross-validation. The white-box approach achieved an AUC of 0.7083 and a classification rate of 0.6473 . The black-box approach achieved an average AUC of 0.6965 and an average classification rate of 0.6421 , as presented in Table 2. It can be seen that the white-box results are slightly better than using cross-validation. However, as previously discussed, white-box statistics (AUC and classification rates) measure data fitting, while cross-validation statistics measure error prediction.

Table 3 shows the AUC, classification rates and computing times for failure detection in joint 6 . Joint 6 has the second largest rate of failures (5.7\%). The anomaly score was estimated using the local variables for joint 6 . Results show that xgboot achieved the best AUC statistic, followed by Random Forests and glmnet. The xgboost achieved the best classification rate, followed by Random Forests and glmnet. The logistic regression achieved the best computing time, followed by xgboot. In general, xgboot, Random Forests and glmnet achieved the best AUC and classification rates.

Table 3: AUC, classification rates and computing times evaluated using 10 runs of the selected classification methods for failure detection in joint 6 . Best results and minimum standard deviations are indicated in bold type.

\begin{tabular}{lrrrrrr}
\hline & \multicolumn{2}{c}{ AUC } & \multicolumn{2}{c}{ Classification rates } & Computing time (min) \\
\cline { 2 - 7 } method & mean & sd & mean & sd & mean & sd \\
\hline glmnet & 0.7432 & 0.0029 & 0.6785 & 0.0031 & 48.4502 & 3.9208 \\
glmNNET350 & 0.7292 & 0.0044 & 0.6613 & 0.0052 & 48.8511 & 14.8300 \\
glmNNET75 & 0.7285 & 0.0047 & 0.6581 & 0.0058 & 13.0907 & 2.6724 \\
logistic & 0.7218 & $\mathbf{0 . 0 0 1 4}$ & 0.6648 & $\mathbf{0 . 0 0 2 4}$ & $\mathbf{0 . 0 1 4 7}$ & $\mathbf{0 . 0 0 3 1}$ \\
Random Forests & 0.7470 & 0.0039 & 0.6846 & 0.0039 & 57.5615 & 1.8703 \\
xgboost & $\mathbf{0 . 7 5 1 6}$ & 0.0072 & $\mathbf{0 . 6 8 8 7}$ & 0.0076 & 0.1463 & 0.0081 \\
\hline
\end{tabular}


Table 4 shows the AUC, classification rates and computing times for failure detection in joint 5 . Joint 5 has the third largest rate of failures $(2.4 \%)$. The anomaly score was estimated using the local variables for joint 5 . Results show that the glmNNET350 model achieved the best AUC statistic, followed by xgboost and glmNNET75. The xgboost achieved the best classification rate, followed by Random Forests and glmNNET350. The logistic regression achieved the best computing time, followed by xgboost.

Table 4: The AUC, classification rates and computing times, evaluated in 10 runs of the selected classification methods for failure detection in joint 5. Best results and minimum standard deviations are indicated in bold type.

\begin{tabular}{lrrrrrr}
\hline & \multicolumn{2}{c}{ AUC } & \multicolumn{2}{c}{ Classification rates } & Computing time (min) \\
\cline { 2 - 6 } method & mean & sd & mean & sd & mean & sd \\
\hline glmnet & 0.7772 & 0.0034 & 0.7075 & 0.0097 & 46.6676 & 2.8222 \\
glmNNET350 & $\mathbf{0 . 8 1 3 4}$ & 0.0044 & 0.7295 & 0.0087 & 79.9149 & 21.4667 \\
glmNNET75 & 0.8118 & 0.0049 & 0.7260 & 0.0085 & 23.3417 & 5.1136 \\
logistic & 0.7118 & 0.0051 & 0.6500 & $\mathbf{0 . 0 0 5 1}$ & $\mathbf{0 . 0 1 6 3}$ & $\mathbf{0 . 0 0 2 4}$ \\
Random Forests & 0.7991 & $\mathbf{0 . 0 0 3 2}$ & 0.7397 & 0.0072 & 48.5733 & 12.7230 \\
xgboost & 0.8124 & 0.0086 & $\mathbf{0 . 7 4 0 4}$ & 0.0075 & 0.1371 & 0.0095 \\
\hline
\end{tabular}

Table 5 shows AUC, classification rates and computing time for failure detection in joint 3 . Joint 3 has the proportion of failures of $1.6 \%$. The anomaly score was estimated using the local variables for joint 3 . Results show that the glmnet model achieved the best AUC statistic, followed by logistic and Random Forests. The glmnet achieved the best classification rate, followed by logistic and Random Forests. The logistic regression achieved the best computing time, followed by xgboost.

Table 5: The AUC, classification rates and computing times, evaluated in 10 runs of the selected classification methods for failure detection in joint 3. Best results and minimum standard deviations are indicated in bold type.

\begin{tabular}{lrrrrrr}
\hline \multirow{2}{*}{ method } & \multicolumn{2}{c}{ AUC } & \multicolumn{2}{c}{ Classification rates } & Computing time (min) \\
\cline { 2 - 6 } & mean & sd & mean & sd & mean & sd \\
\hline glmnet & $\mathbf{0 . 8 6 7 2}$ & 0.0049 & $\mathbf{0 . 8 1 1 1}$ & 0.0068 & 120.5789 & 10.8479 \\
glmNNET350 & 0.8138 & 0.0079 & 0.7535 & 0.0136 & 104.5456 & 18.0655 \\
glmNNET75 & 0.8092 & 0.0071 & 0.7495 & 0.0080 & 36.4443 & 6.7516 \\
logistic & 0.8667 & $\mathbf{0 . 0 0 1 8}$ & 0.8071 & $\mathbf{0 . 0 0 3 2}$ & $\mathbf{0 . 0 1 4 2}$ & $\mathbf{0 . 0 0 1 5}$ \\
Random Forests & 0.8360 & 0.0111 & 0.7879 & 0.0117 & 47.5031 & 2.4083 \\
xgboost & 0.8074 & 0.0123 & 0.7778 & 0.0158 & 0.1385 & 0.0122 \\
\hline
\end{tabular}

Table 6 shows AUC, classification rates and computing time for failure detection in joint 2 . Joint 2 has the proportion of failures of $1.1 \%$. The 
anomaly score was estimated using the local variables for joint 2. Results show that the glmnet model achieved the best AUC statistic, followed by logistic and xgboost. The glmnet achieved the best classification rate, followed by logistic and xgboost. The logistic regression achieved the best computing time, followed by xgboost.

Table 6: The AUC, classification rates and computing times evaluated in 10 runs of the selected classification methods for failure detection in joint 2. Best results and minimum standard deviations are indicated in bold type.

\begin{tabular}{lrrrrrr}
\hline & \multicolumn{2}{c}{ AUC } & \multicolumn{2}{c}{ Classification rates } & \multicolumn{2}{c}{ Computing time (min) } \\
\cline { 2 - 7 } method & mean & $\mathrm{sd}$ & mean & $\mathrm{sd}$ & mean & $\mathrm{sd}$ \\
\hline glmnet & $\mathbf{0 . 8 7 4 5}$ & 0.0094 & $\mathbf{0 . 8 2 9 9}$ & 0.0104 & 218.7813 & 23.7175 \\
glmNNET350 & 0.8229 & 0.0085 & 0.7567 & 0.0123 & 150.7080 & 16.2985 \\
glmNNET75 & 0.8215 & 0.0124 & 0.7567 & 0.0142 & 47.2849 & 14.4059 \\
logistic & 0.8737 & $\mathbf{0 . 0 0 1 5}$ & 0.8179 & $\mathbf{0 . 0 0 6 3}$ & $\mathbf{0 . 0 1 6 8}$ & $\mathbf{0 . 0 0 8 1}$ \\
Random Forests & 0.8534 & 0.0119 & 0.7806 & 0.0101 & 37.4156 & 2.3232 \\
xgboost & 0.8605 & 0.0126 & 0.8134 & 0.0106 & 0.1322 & 0.0098 \\
\hline
\end{tabular}

Table 7 shows the best three classification methods for each joint and each performance measure. Results do not include joint 1, due to the lower rate of failures. In general, there is no single method that achieved the best AUC or classification rate performance for all joints. In general, the blackbox models performed better for larger rate of failure. For lower proportion of failures, the regularized logistic (glmnet) and logistic models performed better. The logistic and xgboost were the fastest methods in all joints. This is because these methods are compiled into lower level programming language, whereas the remaining method uses higher level language. The methods were evaluated using the i7 Intel core and the $\mathrm{R}$ language ( $\mathrm{R}$ Core Team, 2017).

Figure 8 shows HGB cross validation results for failure prediction in joint 5 , in 10 runs. Joint 5 achieved the largest difference, of $9.04 \%$ between the logistic and the best classification rate result, using xgboost. Therefore, the HGB was applied to boost the logistic regression and to identify the additional predictors. Both xgboost and Random Forests were used to boost the logistic regression because they achieved the best classification rate results, as shown in Table 4. Figure 8(a) shows HGB classification rate results using logistic and xgboost. In general, after 20 boosts, the HGB model achieves the same classification rate result obtained using only xgboost. Figure 8(b) shows HGB classification rate results using logistic and Random Forests. In general, after 25 boosts, the HGB model achieves the same classification rate result obtained using only xgboost. Therefore, using xgboost, the HGB 
Table 7: Best classification models for failure detection in joints 2 to 6 . Observed proportion of failures are indicated in parenthesis.

\begin{tabular}{clll}
\hline \multicolumn{1}{c}{ Joint } & \multicolumn{1}{c}{ AUC } & Classification rates & Computing time \\
\hline $2(1.1 \%)$ & glmnet, logistic, xgboost & glmnet, logistic, xgboost & logistic, xgboost \\
\hline $3(1.6 \%)$ & $\begin{array}{l}\text { glmnet, logistic, } \\
\text { Random Forests }\end{array}$ & $\begin{array}{l}\text { glmnet, logistic, } \\
\text { Random Forests }\end{array}$ & logistic, xgboost \\
\hline $4(6.3 \%)$ & $\begin{array}{l}\text { xgboost, Random Forests, } \\
\text { glmnet }\end{array}$ & $\begin{array}{l}\text { xgboost, Random Forests, } \\
\text { glmnet }\end{array}$ & logistic, xgboost \\
\hline $5(2.4 \%)$ & $\begin{array}{l}\text { glmNNET350, xgboost, } \\
\text { glmNNET75 }\end{array}$ & $\begin{array}{l}\text { xgboost, Random Forests, } \\
\text { glmNNET350 }\end{array}$ & logistic, xgboost \\
\hline $6(5.7 \%)$ & $\begin{array}{l}\text { xgboost, Random Forests, } \\
\text { glmnet }\end{array}$ & xgboost, Random Forests & logistic, xgboost \\
& & & \\
\hline
\end{tabular}

requires fewer boosts as compared to Random Forests. Nevertheless, HGB using either xgboost or Random Forests achieves similar final results.

Figure 9 shows the additional predictors selected by xgboost and Random Forests using HGB. Predictors were sorted in decreasing order of feature importance. In general, predictors with larger feature importance are associated with global variables such as production time (p_time), consumed energy (sys_energy) or rate of consumed energy (logSysPtime). Remaining predictors are associate with relative frequency of speed and torque (histogram variables) in different joints. It is worth mentioning that both xgboost and Random Forests rely on random partitions of the predictors. Furthermore, most of the predictors are highly correlated. Therefore, feature importance results may change in different runs.

HGB can also be used to boost a base line model, with a subset of the original data base. This is illustrated in Figure 10. As opposed to using the total of 276 predictors, the HGB was applied using logistic as the base line model and xgboost with a subset of 45 predictors. Local joint predictors and robot operational features were used. Histogram predictors were not included. Results indicate that HGB may improve classification results if a smaller number of predictors is applied. Compared to Figures 8(a) and $8(\mathrm{~b})$, the HGB, using a smaller number of predictors, achieved better classification results. Figure 10(b) shows the xgboost feature importance index. Results show that robot production time (p_time), rate of consumed energy (logSysPtime) and average speed on axis 1 (ax1_avg_speed_rpm) are the main additional predictors for improving failure detection in joint 5 , using a non-linear black-box. 


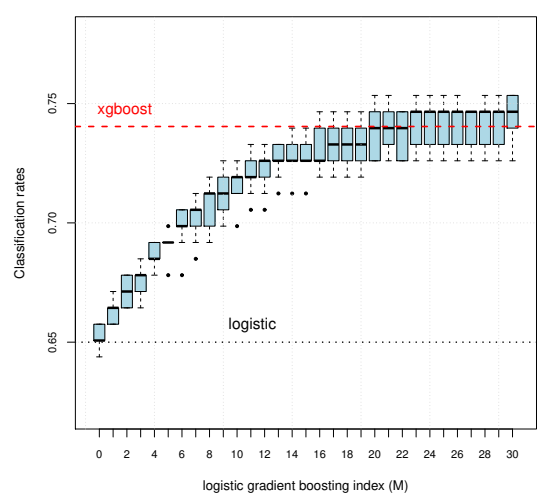

(a) Hybrid gradient boosting with Logistic (b) Hybrid gradient boosting with Logistic and Xgboost.

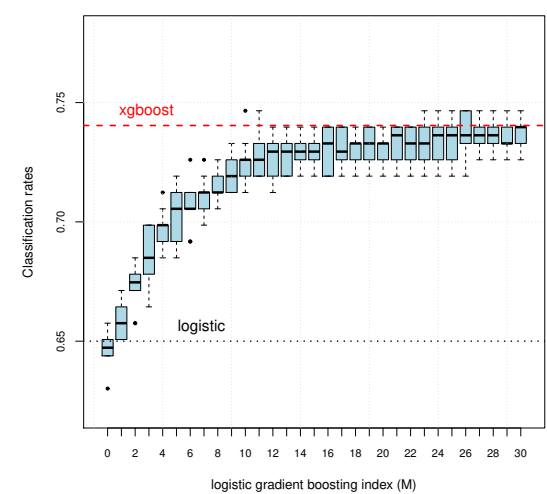

and Random Forests.

Figure 8: Classification rates results using hybrid gradient boosting and logistic regression as the base line model. Original logistic and xgboost results are shown as horizontal lines.

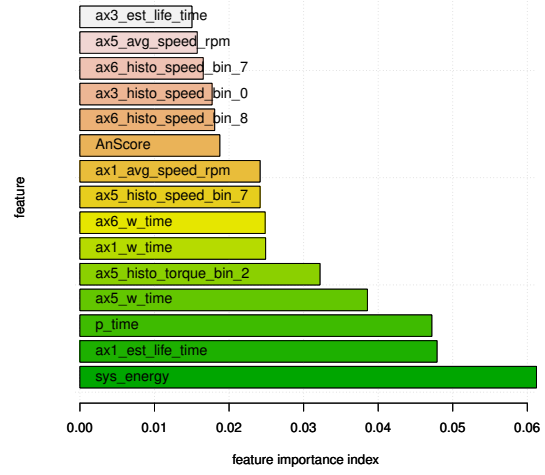

(a) Hybrid gradient boosting with Logistic (b) Hybrid gradient boosting with Logistic and Xgboost.

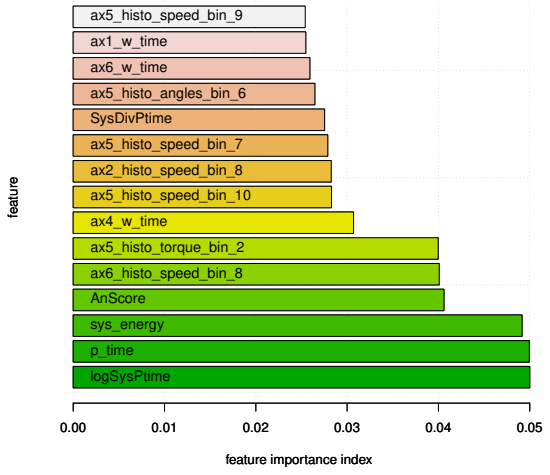

and Random Forests.

Figure 9: Feature importance analysis using hybrid gradient boosting with logistic regression as the base line model and xgboost.

\section{Discussion}

There is a natural scheme for data analysis, which is data consistency analysis followed by data modeling. Data consistency analysis, also known as data cleaning, comprises outlier analysis and statistical description of 


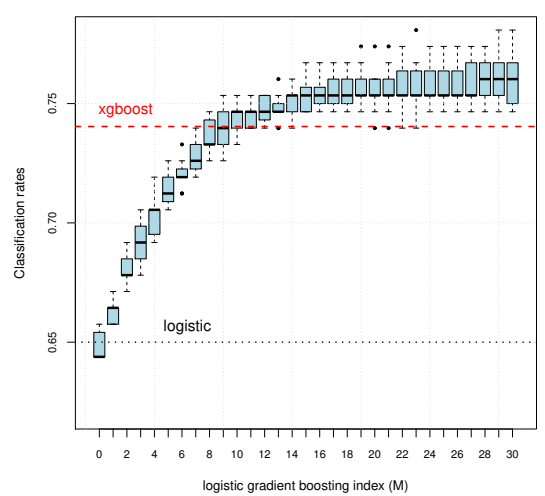

(a) Hybrid gradient boosting with Logistic and Xgboost.

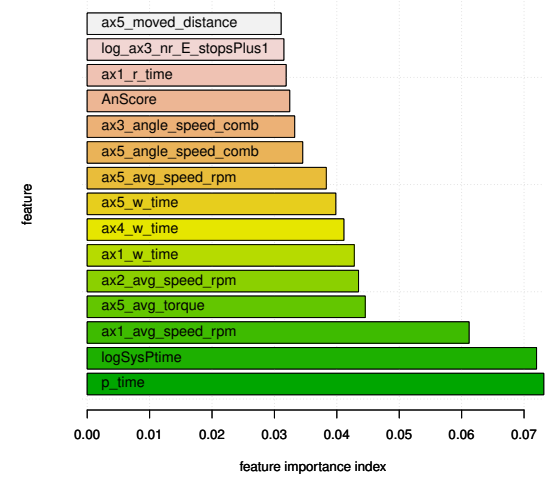

(b) Hybrid gradient boosting with Logistic and Random Forests.

Figure 10: Feature importance analysis using hybrid gradient boosting, logistic regression as the base line model, xgboost and a smaller subset of predictors.

all available variables. Data consistency also includes careful evaluation of the data generation process and checking whether the available information comprises the current beliefs of engineers and analysts with respect to the problem at hand. This is one of the most important steps in data modeling.

Although not reported in this work, the available data set was primarily evaluated using simple descriptive statistics, histograms, correlation matrices, scatter plots, among other exploratory statistical techniques. Furthermore, these results were presented to, and systematically discussed with, engineers and analysists. Future work aims at evaluating additional information in the model. Nevertheless, current findings provided some important insights with respect to failure detection in robotic arms. First, local information is a strong predictor. For instance, the logistic regression achieved a classification cross validation performance of $66.48 \%$ for joint 6 using only local variables, while the xgboost achieved an additional improvement performance of $2.39 \%$ using all 276 variables. For joint 2, glmnet, logistic and xgboost achieved very similar results, providing evidence that failures in joint 2 are driven mostly by local variables. Second, there is evidence that information from different joints improves prediction for a specific joint. For instance, logistic regression classification rates using local information for joint 5 is $65 \%$, whereas xgboost achieves $74 \%$ using information from all joints.

The proposed hybrid gradient boosting model presented very interest- 
ing results by creating a black-box model on top of a white-box model, thereby improving cross-validation performance and separating predictors into white- and black-box contributors. In the present study, both xgboost and random forests achieved best results for joints having large proportions of failures. Furthermore, the HGB results combining standard logistic, Random Forests and xgboost presented promising results. One may claim that the standard logistic regression creates a general classification model, whereas Random Forests and xgboost further improve classification by creating specific partitions of the data set, i.e., local classification models. An interesting finding is the improvement of HGB results if a smaller data set is applied in the boosting procedure. This might be related to the tree-based models. As indicated by Liu et al. (2008), the isolation forest performs better with a smaller number of predictors. This may be the case for both the xgboost and random forests models.

A critical point, in assessing classification performance, is the selection of the appropriate statistical measure. Although AUC is a common choice for evaluating classification models, in practice, predictive classification error or classification rate is a suitable choice. As previously discussed, most classification models estimate the chance of failure, from which the analyst must decide whether the failure will happen based on a threshold. The AUC statistic is robust for the threshold choice. However, results show major differences between AUC and classification rate. For instance, the xgboost AUC result for joint 6 is $75.16 \%$, whereas for the classification rate it is $68.87 \%$. Therefore, it worth mentioning that AUC should not be used as a proxy for predictive classification rates. Results show that AUC and classification rates are different classification measures, which may or may not achieve similar results.

Regarding the logistic regression results, one may claim that white-box models, such as the logistic regression, do not require cross-validation evaluation because they rely on consistent statistical theory/statistical inference. In general, this statement is true. Nonetheless, results show that, in general, the white-box model (logistic) resulted in slightly larger prediction errors. However, for joint 2, both glmnet and logistic achieved the best predictive results, as shown in Table 6 . Furthermore, joint 2 has a lower rate of failures (1.1\%). This may suggest that, for rare failure events, a simpler white-box model may outperform sophisticated black-box models.

Black box models may produce more reliable information about the structure of the relationship between inputs and outputs than white box models, mostly because they do not assume any prior parametric structure about the underlying structure of the data. On the contrary, white box mod- 
els produce a simple and understandable picture of the relationship between the input variables and the output variable. For example, logistic regression is frequently used in classification problems because it produces a linear combination of the variables with parameters that indicate the variables' importance.

It is worth mentioning that the logistic model can be estimated using a black-box approach. For instance, one may evaluate hundreds or thousands of different logistic models and select the few models with the best error prediction. For example, assuming a fixed number of variables, say 5 variables, and assuming a database with only 50 variables, there are 2,118,760 different models. In this case, a white-box approach saves time and may achieve reasonable results.

\section{Conclusion}

The present work has proposed classification models for failure detection in industrial robotic arms, using statistical and machine learning methods. The standard logistic regression model was adjusted using the white-box approach, i.e., with the available data set and selecting variables using statistical inference. In addition, four machine learning models were adjusted using the black-box approach, i.e., with all available variables and crossvalidation performance. Finally, a hybrid approach, using both white- and black-box models, was proposed and evaluated. The white-box component indicates the importance of each variable, whereas the black-box component further improves the error prediction.

As expected, the different methods performed differently for the different joints. In general, simple methods achieved better performance for joints with lower rates of failures using local joint information. Furthermore, using anomaly scores as an additional variable helped to improve classifications.

It is worth mentioning that the construction of white-box, black-box and hybrid models for failure detection must rely on data analysis and technical knowledge of the production process. Therefore, decisions, with respect to selection of variables for anomaly score estimation, were based on local joint information previously discussed with engineers and analysts.

In general, the development of failures and wear is dependent on the accumulated usage of the robot. By including several sensors and providing different measurements per robot, a natural step is to correlate these

measurements with the failures and, therefore, develop predictive failure classification models. The current database provided some very interesting 
insights into the main drivers of failures in robotic arms. However, we believe more data are required in order to continue to improve the classification results.

\section{Acknowledgements}

The authors thank CISB Swedish-Brazilian Research and Innovation Center, Saab AB and the VINNOVA sponsored Competence Center LINK-

SIC for financial support. We also appreciated fruitful comments and discussions of the anomaly study group coordinated by Professors Eric Frisk and Mattias Krysander.

\section{References}

Altman, D. G., 1994. Statistics notes: Diagnostic tests 1: sensitivity and specificity. British Medical Journal 308, 1552.

Bradley, A. P., 1997. The use of the area under the roc curve in the evaluation of machine learning algorithms. Pattern recognition 30 (7), 1145-1159.

Breiman, L., 2001. Random forests. Machine learning 45 (1), 5-32.

Breiman, L., 2017. Classification and regression trees. Routledge.

Breiman, L., et al., 2001. Statistical modeling: The two cultures (with comments and a rejoinder by the author). Statistical science 16 (3), 199-231.

Chen, T., He, T., Benesty, M., Khotilovich, V., Tang, Y., 2018. xgboost: Extreme Gradient Boosting. R package version 0.6.4.1.

URL https : //CRAN.R-project.org/package=xgboost

Christopher, M. B., 2011. Pattern Recognition and Machine Learning. Springer-Verlag New York.

Dobson, A. J., Barnett, A., 2008. An introduction to generalized linear models. CRC press.

Domingues, R., Filippone, M., Michiardi, P., Zouaoui, J., 2018. A comparative evaluation of outlier detection algorithms: Experiments and analyses. Pattern Recognition 74, 406-421. 
Fan, S., Zhang, Y., Zhang, Y., Fang, Z., 2017. Motion process monitoring using optical flow-based principal component analysis-independent component analysis method. Advances in Mechanical Engineering 9 (11), $1-12$.

Fawcett, T., 2004. Roc graphs: Notes and practical considerations for researchers. Machine learning 31 (1), 1-38.

Friedman, J., Hastie, T., Tibshirani, R., 2001. The elements of statistical learning. Vol. 1. Springer series in statistics New York.

Friedman, J., Hastie, T., Tibshirani, R., 2010. Regularization paths for generalized linear models via coordinate descent. Journal of statistical software 33 (1), 1-22.

Friedman, J. H., 2001. Greedy function approximation: a gradient boosting machine. Annals of statistics, 1189-1232.

Gybenko, G., 1989. Approximation by superposition of sigmoidal functions. Mathematics of Control, Signals and Systems 2 (4), 303-314.

Halder, B., Sarkar, N., 2009. Analysis of order of redundancy relation for robust actuator fault detection. Control engineering practice 17 (8), 966973.

Hoerl, A. E., Kennard, R. W., 1970. Ridge regression: Biased estimation for nonorthogonal problems. Technometrics 12 (1), 55-67.

Huang, G.-B., Zhu, Q.-Y., Siew, C.-K., 2006. Extreme learning machine: theory and applications. Neurocomputing 70 (1-3), 489-501.

Khalastchi, E., Kalech, M., Rokach, L., 2017. A hybrid approach for improving unsupervised fault detection for robotic systems. Expert Systems with Applications 81, 372-383.

Liu, F. T., Ting, K. M., Zhou, Z.-H., 2008. Isolation forest. In: Data Mining, 2008. ICDM'08. Eighth IEEE International Conference on. IEEE, pp. 413422.

Ma, H.-J., Yang, G.-H., 2016. Simultaneous fault diagnosis for robot manipulators with actuator and sensor faults. Information Sciences 366, 12-30.

Makridakis, S., Spiliotis, E., Assimakopoulos, V., 2018. Statistical and machine learning forecasting methods: Concerns and ways forward. PloS one $13(3), 1-26$. 
Montgomery, D. C., 2007. Introduction to statistical quality control. John Wiley \& Sons.

Montgomery, D. C., Peck, E. A., Vining, G. G., 2012. Introduction to linear regression analysis. Vol. 821. John Wiley \& Sons.

Murthy, S. K., 1998. Automatic construction of decision trees from data: A multi-disciplinary survey. Data mining and knowledge discovery 2 (4), $345-389$.

Nelder, J. A., Baker, R. J., 1972. Generalized linear models. Wiley Online Library.

Puggini, L., McLoone, S., 2018. An enhanced variable selection and isolation forest based methodology for anomaly detection with oes data. Engineering Applications of Artificial Intelligence 67, 126-135.

R Core Team, 2017. R: A Language and Environment for Statistical Computing. R Foundation for Statistical Computing, Vienna, Austria.

URL https://www.R-project.org/

Shahriari-kahkeshi, M., Sheikholeslam, F., Askari, J., 2015. Adaptive fault detection and estimation scheme for a class of uncertain nonlinear systems. Nonlinear Dynamics 79 (4), 2623-2637.

Tibshirani, R., 1996. Regression shrinkage and selection via the lasso. Journal of the Royal Statistical Society. Series B (Methodological), 267-288.

Zou, H., Hastie, T., 2005. Regularization and variable selection via the elastic net. Journal of the Royal Statistical Society: Series B (Statistical Methodology) 67 (2), 301-320. 